\title{
Fingerprint Study of Curcuma xanthorrhiza Rhizome by High Performance Thin Layer Chromatography (HPTLC)
}

Penulis

Afiliasi
Tina Wikara ${ }^{*}$, Anny Sulistiowaty, Sri Murhandini and Tepy Usia

Research Center for Drug and Food-BPOM RI

Kata Kunci
$\Rightarrow$ Curcuma xanthorrhiza
$\quad$ rhizome
$\Rightarrow$ curcumin
$\partial$ fingerprint
$\Rightarrow$ HPTLC

Diterima 7 September 2015 Direvisi 14 Juli 2016 Disetujui 23 September 2016

*Corresponding author Tina Wikara

Research Center for Drug and Food-BPOM RI Jl. Percetakan Negara No. 23 Jakarta Pusat 10560 Email: tina_wikara@yahoo.com

\section{Abstract}

The rhizome of Curcuma xanthorrhiza Roxb is intensively used in Indonesia as traditional medicine. It is widely used for hepatoprotective and anti inflammatory activities. To ensure the quality of its extract, we have studied the fingerprint or phytochemical analysis. This research was aimed to produce a chromatogram profile of the rhizome by HPTLC. The HPTLC fingerprint chromatogram of $C$. xanthorrhiza rhizome was performed using HPTLC plate of silica gel $60 \mathrm{~F}_{254}$ as the stationary phase and chloroform-methanol (97:3) as the mobile phase. Spot detection was carried out by TLC photo documentary system at 254 and $366 \mathrm{~nm}$ and TLC scanner at $427 \mathrm{~nm}$. The developed method was validated according to $\mathrm{ICH}$ guidelines by determination of specificity and precision. We found that the specifity and precission of the method were met the acceptance criteria. In conclusion, the developed method is valid and could be used for quality control and standardization of herbal medicine containing $C$. xanthorrhiza rhizome.

\section{Introduction}

Indonesia is an endowed country with a variety of medicinal plants with strong potential for a therapeutic application. Curcuma xanthorrhiza is one of the most popular medicinal herbs where the rhizome is intensively used in Indonesian traditional medicine for hepatoprotective and anti inflammation activities (Li et al. 1995). Two major groups of compounds that have been known to be responsible for the pharmacological activities of Curcuma are curcuminoid and volatile oil (Syu et al. 1998).

The efficiency of drug therapy is highly dependent on the use of proper and originality of raw materials. Therefore, the guarantee of safety, quality and efficacy of medicinal plants and herbal products has now become a major and important issue so that the plant material required standardization. In recent years, progress in chromatographic and spectral fingerprint has played an important role especially in the quality control of complex herbal medicines (Yamunadevi et al. 2011).

Fingerprint analysis by high performance thin layer chromatography (HPTLC) has become an effective and powerful tool for the estimation of chemical and biochemical markers. HPTLC is becoming a routine analytical technique because of some advantage 
including the small amount of mobile phase required the speed of the method, and the possibility of analysis of several samples simultaneously (i.e. on the same plate), unlike HPLC. It thus reduces analysis time and cost per analysis. Cloudy samples and suspensions can also be analyzed directly by HPTLC (Ali et al. 2007). Automatic sample application is possible and repeated scanning can be performed on the same plate, so scanning conditions can be changed.

The rhizomes of $C$. xanthorrhiza and turmeric (Curcuma longa) are generally having a yellow or orange color due to the present of curcuminoids. This study aims to obtain a fingerprint/ chromatogram profile of $C$. xanthorrhiza as a basic for standardization to ensure the safety and quality of $C$. xanthorrhiza using HPTLC technique. This method could be used for identification, differentiation and authentication of raw and extract of $C$. xanthorrhiza.

\section{Methodology Material}

The rhizomes of $C$. xanthorrhiza were collected from 3 (three) different locations: Bandung, Solo and Yogyakarta. Samples of turmeric were colected from Bandung. It were determined in Herbarium Bogoriense, Research Centre for Biology, Indonesian Institute of Science, Bogor, Indonesia. The reference standard used in this research was curcuminoid (Sigma-Aldrich, Singapore, Singapore) and the solvent were chloroform and methanol (Merck. Darmstadt, Germany). High Performance Thin Layer Chromatography (HPTLC) silica gel glass plate $\mathrm{F}_{254}(20 \times 10 \mathrm{~cm})$ were used for HPTLC fingerprint analysis.

\section{Equipment}

HPTLC fingerprint was carried out using Linomat 5 TLC Spotter (Camag, Muttenz, Switzerland), twin through glass chamber $20 \times 10 \mathrm{~cm}$, TLC Scanner 3 (Camag, Muttenz, Switzerland), TLC Documentation System Reprostar3 (Camag, Muttenz, Switzerland).

\section{Methods}

\section{Method Development}

Standard Solution

Standard solution was prepared by dissolving curcuminoid (1 mg) in $1 \mathrm{~mL}$ of methanol.

Test Solution

All powder sample were extracted using methanol as solvent. Approximately $0.1 \mathrm{~g}$ dried powder of C. xanthorrhiza (Bandung, Solo and Yogyakarta) and turmeric was put into $2 \mathrm{~mL}$ centrifuge tube, added $1 \mathrm{~mL}$ methanol then sonicated at $60{ }^{\circ} \mathrm{C}$ for 5 minutes. The mixture was centrifuged at $4000 \mathrm{rpm}$ for 5 minutes. Supernatant was used as a test solution. This procedure was repeated 6 times.

HPTLC fingerprint Analysis

Test and standard solution were applied to plates using Linomat 5 TLC Spotter. Five microliters of each test solution were spotted as a band width of 7.0 $\mathrm{mm}$ on an HPTLC silica gel glass plate $F_{254}(20 \times 10 \mathrm{~cm})$. The following conditions were employed: distance from bottom plate $10 \mathrm{~mm}, \mathrm{x}$-potion from $1^{\text {st }}$ track $12 \mathrm{~mm}$. HPTLC plate was eluated in chloroform-methanol (97:3). Linear ascending development was carried out in $20 \times 10 \mathrm{~cm}$ twin trough glass chamber (CAMAG) saturated with the mobile phase $(20 \mathrm{~mL}$ back side and $10 \mathrm{~mL}$ front side) and filter paper at back side for 20 minutes. The length of each chromatogram run was 85 $\mathrm{mm}$. After developing, the TLC plate was dried using an air dryer. Densitometry scanning was performed on Camag TLC scanner 3 in the reflectance-absorbance mode at $427 \mathrm{~nm}$. Densitometry of the TLC chromatogram was carried out on Camag reprostar 3.

Detection

The dried plate was observed under UV 254 and $366 \mathrm{~nm}$ to visualize the chromatograms, and it were documented by Photo Documentary System (Camag, Muttenz, Switzerland) under UV 254 and $366 \mathrm{~nm}$ and scanned by TLC Scanner (Camag, Muttenz, Switzerland) at $427 \mathrm{~nm}$.

\section{Validation Parameters}

Suitability System Test

Standard solution and test solutions were made, then applicated and developed as in 1.c. After developed, the plate was detected as i 1.d. Retention Factor (Rf) and average $\mathrm{Rf}$ of curcumin spots were specified by TLC Scanner (Camag, Muttenz, Switzerland). Relative Standard Deviation (RSD) of 6 curcumin spots was Calculated. The acceptance criteria was RSD $\leq 2 \%$

Specificity

Standard solution and test solutions were made, then applicated and eluated as in 1.c. After developed, the plate was detected as in 1.d. The spectrum of curcumin in the standard solution and test solution were observed. The acceptance criteria of the curcumin 
spectrums in the standard solution and test solution must be the same.

\section{Precision}

Standard solution and test solutions made, then applicated and developed as in 1.c. After developed, the plate was detected as in 1.d. Rf, average Rf, and RSD of curcumin spots were specified. The acceptance criteria of precision was RSD $\leq 2 \%$. And the acceptance criteria of intermediate precision was RSD $\leq 5 \%$.

\section{Result}

TLC fingerprint analysis is one of the techniques frequently used for evaluation the quality of herbal medicines (Li et al. 1995). Chromatogram/fingerprint profile of $C$. xanthorrhiza had been made using three samples from three locations: Bandung, Solo and Yogyakarta. The rhizome had been authenticated at Research Center for Biology - Indonesian Institute of Sciences. The results showed that the plant used was $C$. xanthorrhiza. This study used curcuminoid as reference standard which was a major compound of $C$. xanthorrhiza (Syu et al. 1998).

C. xanthorrhiza and turmeric is closely related species belongs to the Zingiberaceae family, so they have similarity on the appearance especially in powdered form and chemicals composition but posses different pharmacological activities, so it can be sources for adulteration in the herbal medicines product containing one of two rhizomes (Rafi et al. 2011). TLC fingerprint method for identification, differentiation and authentication of two rhizomes $C$. xanthorrhiza and Curcuma longa have been developed using HPTLC technique.

Chromatographic condition such as, mobile phase have been optimized firstly before applied to

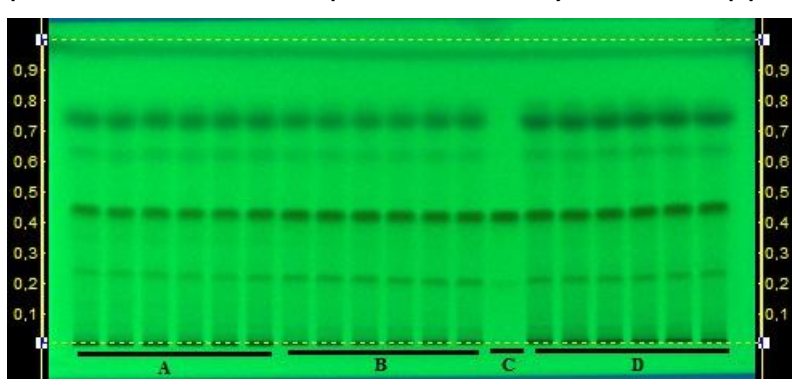

validation. Extraction was done in methanol. Methanol is a polar solvent that could attract curcumin and another polar compound in the rhizome of $C$. xanthorrhiza and turmeric. Fingerprint analysis was conducted using HPTLC silica $\mathrm{F}_{254}$ as a stationary phase and chloroform-methanol (97:3) as a mobile phase which was recorded by photo documentary system at UV 254 and $366 \mathrm{~nm}$ (can be seen on .). The fingerprint of $C$. xanthorrhiza under UV $254 \mathrm{~nm}$ showed spot at a range 0.07-0.78. All samples display a green, blue, red and yellow spot at an approximate $\mathrm{Rf}$ value between 0.02-0.85 under UV $366 \mathrm{~nm}$. The $\mathrm{Rf}$ values of Curcuminoid were investigated as shown in fig 1 . By HPTLC, the Rf value of curcuminoid was confirmed to be curcumin, demethoxycurcumin, and bisdemethoxycurcumin. The average $\mathrm{Rf}$ values of curcumin, demethoxycurcumin, and bisdemethoxycurcumin on mobile phase chloroformmethanol (97:3) was $0.47 ; 0.23$ and 0.19 viewed under UV $366 \mathrm{~nm}$.

From TLC photo documentary System at $\lambda 254$ and $366 \mathrm{~nm}$ (Fig. 1) and TLC scanner at $\lambda 427 \mathrm{~nm}$ (Fig.2), it were found a major peak in the curcuminoid extracts of all samples curcumin. The other two minor peaks were demethoxycurcumin, and bisdemethoxycurcumin. It was showed that all samples from Bandung, Solo and Yogyakarta contained curcumin as a major compound. When compared to its intensity, the curcumin spot from Yogyakarta was the biggest than two others. It means that the highest curcumin content of the three samples was sample from Yogyakarta. While the third chromatogram profiles were similar but there were a little difference in the number of spots and spot intensity. Wavelength at $\lambda \max 427 \mathrm{~nm}$ which was used to analyze each curcuminoids content in the samples is the same

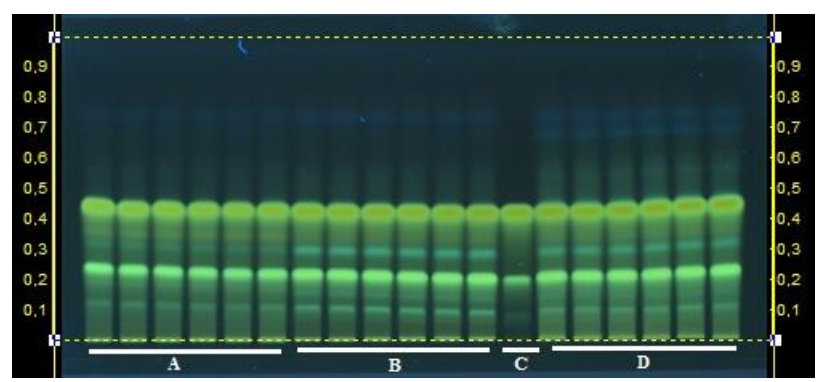

Figure 1. HPTLC fingerprint of C. Xanthorrhiza from various locations: A) Bandung; B) Solo; C) reference standard; D) Yogyakarta. Stationary phase: HPTLC silica F254; Solvent system: Chloroform-methanol (97:3); detection: (a) UV 254; (b) UV $366 \mathrm{~nm}$ 
wavelength recommended by Indonesian Herbal Pharmacopoeia (Kementrian Kesehatan RI 2009).

The system suitability test (SST) was carried out by doing 6 replication samples. The result showed that the RSD values of SST test were below $2 \%$ so all of the suitability system test meets the acceptance criteria, i.e RSD values of 6 sample replicates is less than $2 \%$.

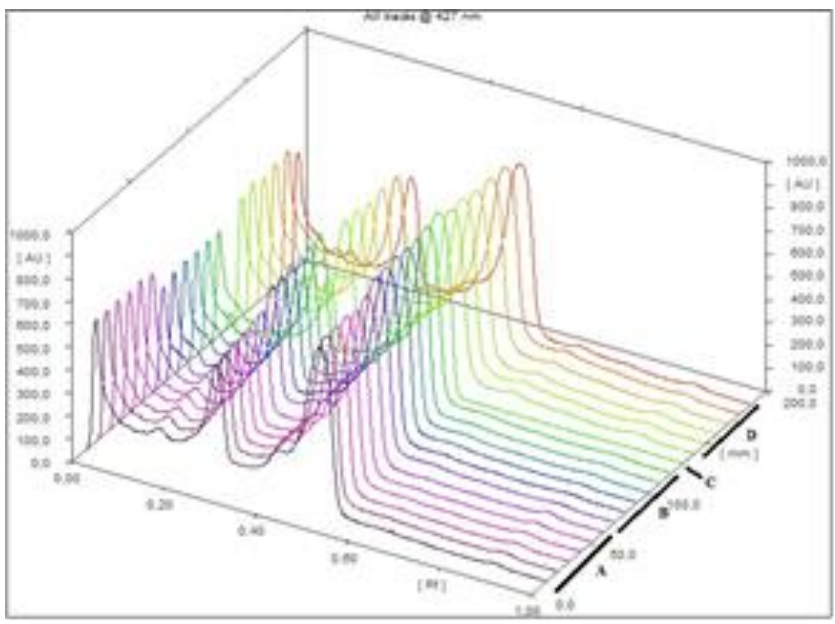

(a)
Therefore, system suitability test results met the requirements.

Specificity test resulted that the curcumin spectra of standard was same as with the spectra of the spot that suggested as curcumin in test solution from Bandung, Solo and Yogyakarta (Fig.3). It was concluded that these spots were curcumin and meet

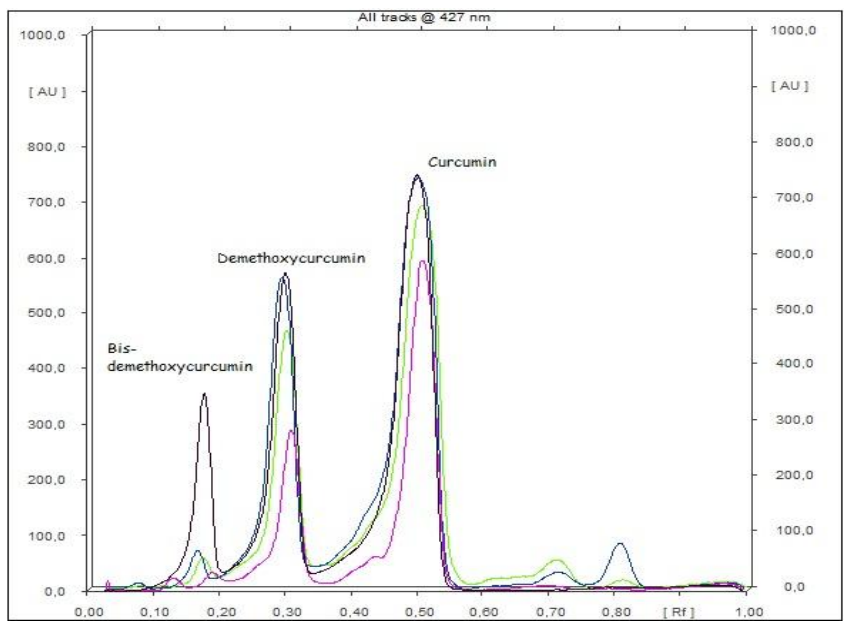

(b)

Figure 2. Chromatogram profile of $C$. Xanthorrhiza from various locations: A) Bandung; B) Solo; C) reference standard; D) Yogyakarta. Detection: $427 \mathrm{~nm}$

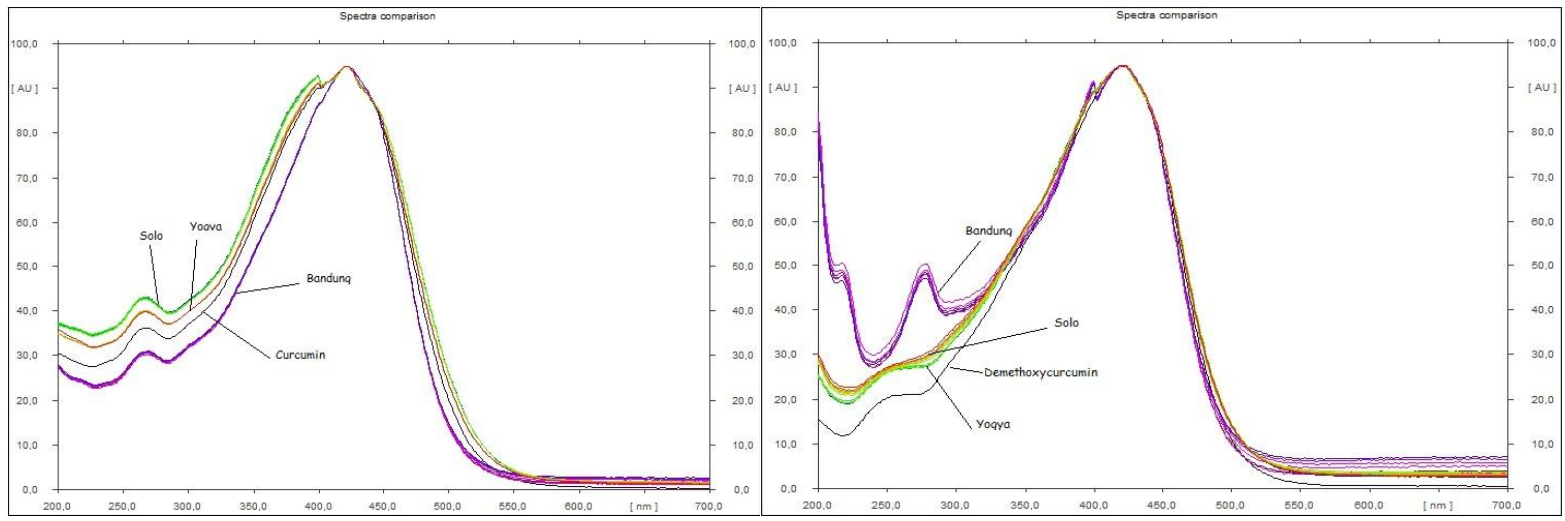

(a)

(b)

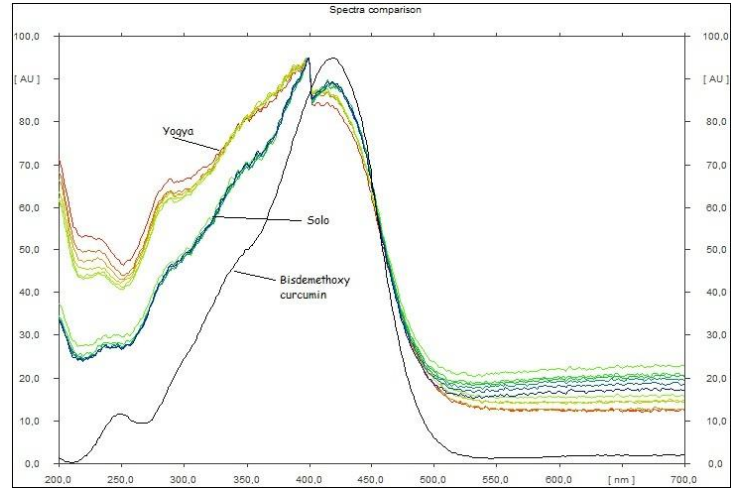

(c)

Figure 3. Spectrum of Standard and Test Solution with mobile phase chloroform-methanol (97:3): (a). Curcumin; (b). Demethoxycurcumin; (c). Bis-demethoxycurcumin 
the specificity test acceptance criteria. The spectra was observed by TLC scanner at $\lambda 427 \mathrm{~nm}$. The curcumin spectra of standard and test solution can be seen in Figure 3.

The intermediate precision was carried out by doing each of 6 replication samples on 2 diffrent plates. The acceptance criterias of intermediate precision were RSD of 6 replication on a plate $\leq 2 \%$ and RSD between 2 plates $\leq 5 \%$. The result showed that the RSD values of all plates tested were less than $2 \%$.

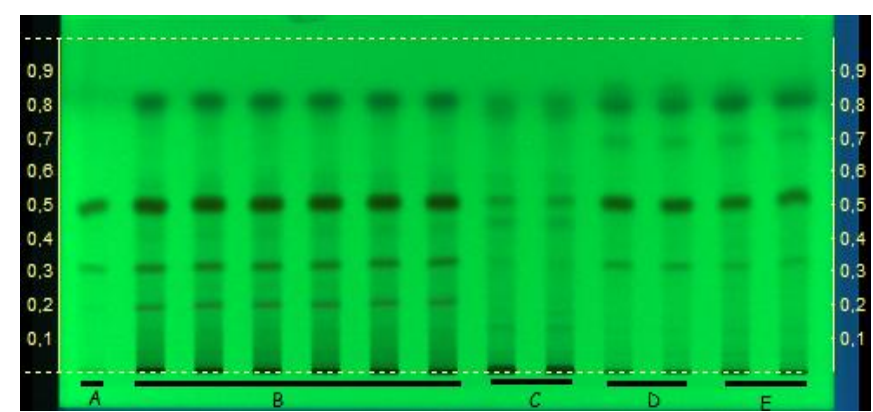

(a)
Figure 4 displays fingerprint chromatograms for C. xanthorrhiza and turmeric viewed under UV (254 and $366 \mathrm{~nm}$ ). The fingerprints of turmeric under UV 254 and $366 \mathrm{~nm}$ clearly showed 4 strong spot at a range $0.19-0.80$, three yellow spot represent curcumin, demethoxycurcumin and bisdemethoxycurcumin. The $\mathrm{Rf}$ values corresponding to $0.47 ; 0.29$ and 0.19 , respectively. The HPTLC fingerprints of $C$. xanthorrhiza give 2 strong spot at $\mathrm{Rf} 0.48$ and 0.82 and 4 weak absorbed weak spot at range Rf 0.12-0.69 under UV

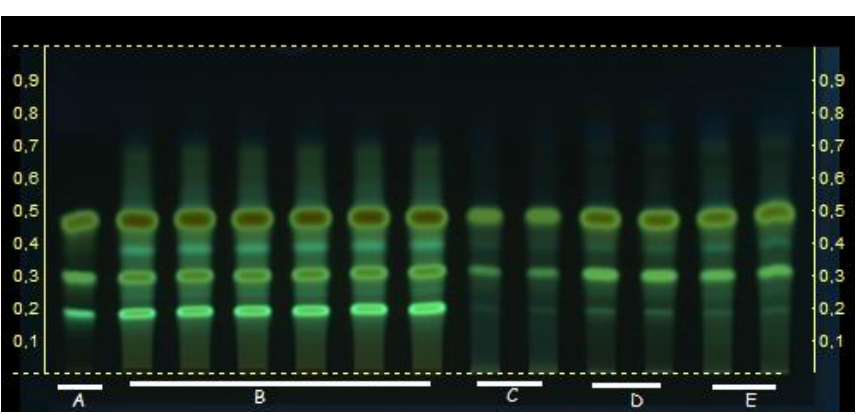

(b)

Figure 4. HPTLC fingerprint of C. Xanthorrhiza from various locations and turmeric: A) Curcuminoid; B) Turmeric; C) Bandung; D) Solo; E) Yogyakarta. Stationary phase: HPTLC silica F254; Solvent system: Chloroform-methanol (97:3); detection: (a) UV 254; (b) UV $366 \mathrm{~nm}$

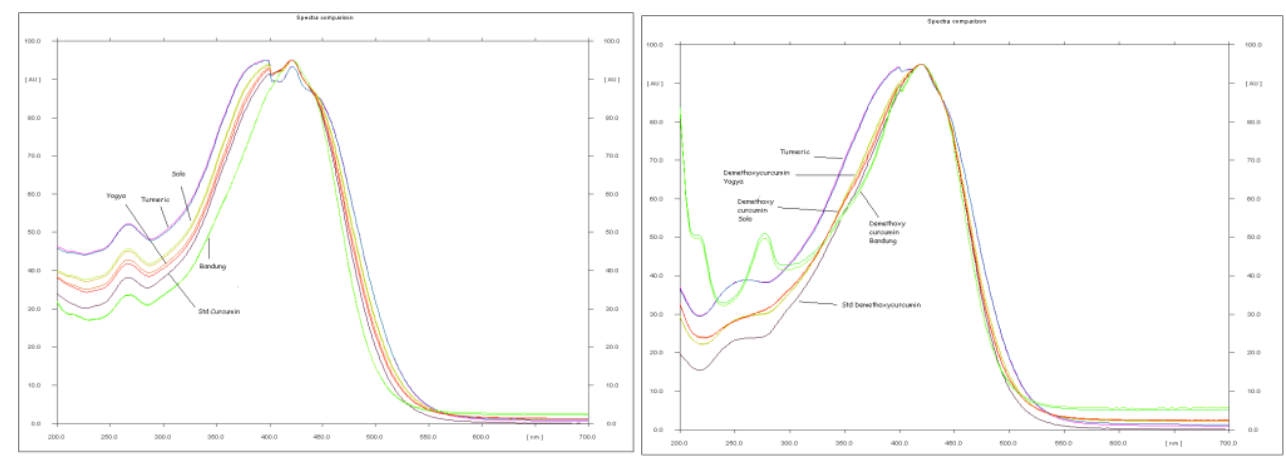

(b) (b)

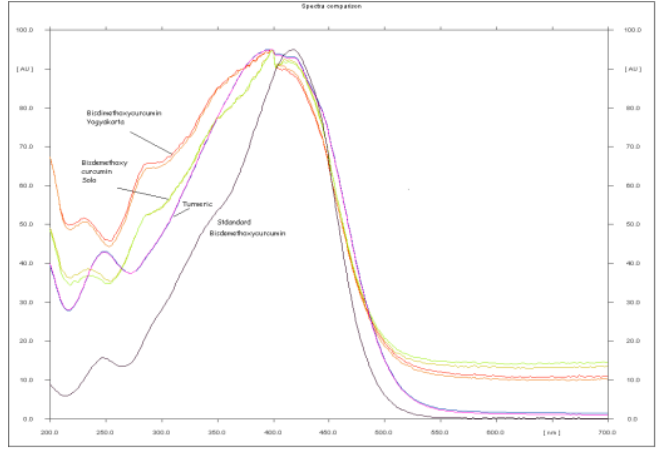

(c)

Figure 5. Spectrum of Standard of Curcuminoid and Test Solution (C.xanthorrhiza and turmeric) with mobile phase chloroform-methanol (97:3): (a). Curcumin; (b). Demethoxycurcumin; (c). Bisdemethoxycurcumin 
$254 \mathrm{~nm}$. The Rf values corresponding to 0.29 and 0.47 represent demethoxycurcumin and curcumin under $366 \mathrm{~nm}$ and a weak absorbed spot at 0.19 represent bisdemethoxycurcumin. Turmeric and C. xanthorrhiza can be differentiating with a 2 marker strong spot at Rf 0.29 and 0.47 respectively. C. xanthorrhiza has a spot at Rf 0.19 but only a weak spot.

Specificity test for C. xanthorrhiza and turmeric resulted that the curcumin spectra of standard was same as with the spectra of the spot that suggested as curcumin in test solution (C. xanthorrhiza and turmeric) (Fig.5). It was concluded that these spots were curcumin and meet the specificity test acceptance criteria. The spectra was observed by TLC scanner at $\lambda$ $427 \mathrm{~nm}$. The curcumin spectra of standard and test solution can be seen in Figure 5. Overall, validation parameters tested in this study had met the requirements so that the method of determining the profile chromatogram profiles/fingerprints C. xanthorrhiza was valid.

\section{Conclusion}

C. xanthorrhiza grown in different parts of Indonesian contains different amounts of various curcuminoid. Therefore, for obtaining the high yield of certain curcuminoid, the area for plant collection should be considered. Based on the results, analytical method validation for the determination of fingerprint of $C$. xanthorrhiza was valid. The results showed that the curcumin spectrum of the standard solution and test solution had appropriate acceptance criteria of specificity, as well as for precision and intermediate precision. In conclusion, the method was valid and it would provide a useful guidance for the quality control and standardization of curcuminoid extracts on the $C$. Xanthorrhiza used in pharmaceutical products and cosmetics.

\section{References}

Ali J, Ali Y, Sultana S, Baboota S, Faiyaz S. 2007. Development and validation of a stabilityinduced HPTLC method for analysis of antitubercular drugs. Acta Chromatographica 18:168-179.

Li, SC, Lin CC, Lin YH, Supriyatna S, Teng CW. 1995. Protective and therapeutic effect of Curcuma xanthorrhiza on hepatotoxin-induced liver damage. American Journal of Chinese Medicine. 23: 243-254.

Menteri Kesehatan Republik Indonesia Farmakope Herbal Indonesia Edisi Pertama, Lampiran Keputusan Menteri Kesehatan No 261/Menkes/SK/IV/2009. 2009.

Rafi M, Eti R, Ali M, Latifah KD. 2011. Differentiation of Curcuma longa, Curcuma xanthorrhiza and Zingiber cassumunar by Thin Layer Chromatography Fingerprint Analysis. Indonesian Journal of Chemistry. 11(1):71-74.

Syu WJ, Shen CC, Don MJ, Ou JC, Lee GH, Sun CM. 1998. Cytotoxycity of curcuminoids and some novel compounds from curcuma zedoria. Journal of Natural Products. 61: 1531-1534.

Yamunadevi M, Wesely EG, Johnson MA. 2011. A chromatographic study on the glycosides of Aerva lanata L. Chinese Journal of Natural Medicines. 9: 210-214. 